\title{
The effect of ultrasound-guided popliteal block combined propofol target-controlled infusion on postoperative pain control after ankle \& foot surgery
}

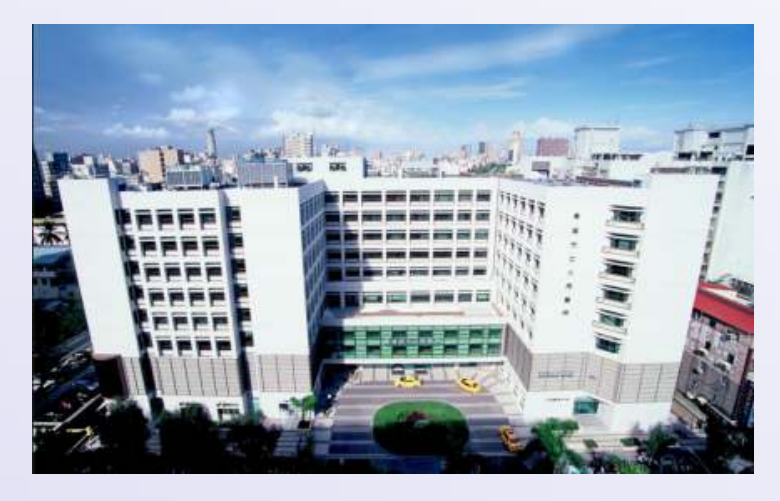

\author{
Authors: Jui-Mei Huang ${ }^{1}$, Yu-Chun Chen ${ }^{1}$, Ya-Ping Chuang ${ }^{1}$, I-Cheng Lu 2 \\ Affilitations:1 Department of Anesthesiology, Kaohsiung Municipal Ta-Tung Hospital,KMU \\ 2 Department of Anesthesiology, Kaohsiung Municipal Hsiao-Kang Hospital, \\ Kaohsiung Medical University (KMU),Kaohsiung, Taiwan
}

\section{Background and Goal of Study:}

Patients usually experience a moderate to severe postoperative pain and require multiple doses of parenteral analgesics after foot and ankle surgery. Popliteal sciatic block (PSB) could provide sufficient anesthesia and reduces postoperative pain. Moreover, it may also avoid complications associated with general anesthesia and neuraxial block such as dental injury or urinary retention. The purpose of this study is to determine the effectiveness of PSB plus sedation on ankle and foot surgery when compared to general anesthesia plus opioids.

\section{Materials and Methods:}

Patients were randomly allocated into PSB group (popliteal sciatic block with propofol sedation) and GA group (general anesthesia). The patients in PSB group $(n=32)$ received intravenous midazolam $2 \mathrm{mg}$ followed by ultrasound-guided popliteal sciatic nerve block with $0.5 \%$ ropivacaine $40 \mathrm{ml}$ at induction room. Propofol target-controlled infusion (TCl) was also kept in PSB group with effect concentration (2.0-3.0 ug/ml) to provide moderate sedation during entire operation. The patients in GA group $(n=35)$ were induced with propofol 2-3 mg. $\mathrm{kg}^{-1}$, followed with sevoflurane inhalation. Laryngeal mask airway was inserted after achieving adequate anesthetic depth, and sevoflurane (2-4\%) was used for intraoperative maintenance. Intraoperative opioids (morphine or fentanyl) was delivered for both groups when necessary. The demographic data, anesthetic parameters, and postoperative recovery (pain intensity, adverse events, analgesics use and satisfaction) were all recorded.

\section{Results and Discussion:}

Patient characters did not differ significantly between groups (Table 1). In PSB group, less patients required intra-operative opioids when compared to GA group (15/32, $47 \%$ vs. $35 / 35$, $100 \%, p<0.001$ ) (Table 2). Postoperative pain intensity was significantly lower in PSB group than GA group when arrival at post-anesthesia care unit $(2.0 \pm 1.8$ vs. $3.3 \pm 1.4, p<0.01)$. In postoperative anesthesia care unit (PACU), less patients required postoperative opioids in PSB group than GA group (9/31, 29\% vs. $27 / 35,77 \%, p<0.001)$. Patients in PSB group depicted better postoperative recovery profile than GA group. Less postoperative nausea vomiting was noted in PSB group when compared to GA group $(5 / 31,16 \%$ vs. $15 / 37,43 \%$, p=0.03) (Table 3).

\section{Conclusion:}

Ultrasound-guided popliteal sciatic block reduced postoperative pain and adverse events in patients undergoing ankle and foot surgery than general anesthesia.

Table 1. Patient characteristics between groups

\begin{tabular}{llll}
\hline & PSB group $(\mathrm{n}=32)$ & GA group $(\mathrm{n}=35)$ & P value \\
\hline Gender (M:F) & $4: 28$ & $9: 26$ & 0.17 \\
Age (yr) & $50.4 \pm 16.7$ & $46.9 \pm 15.1$ & 0.37 \\
Weight $(\mathrm{kg})$ & $60.9 \pm 12.6$ & $61.1 \pm 10.3$ & 0.97 \\
Height $(\mathrm{cm})$ & $157.9 \pm 8.3$ & $160.5 \pm 8.9$ & 0.23 \\
ASA I/II/III(n) & $7 / 21 / 4$ & $6 / 23 / 6$ & 0.81 \\
Anesthesia period (min) & $63.9 \pm 19.2$ & $61.9 \pm 15.8$ & 0.65 \\
Operation period (min) & $40.3 \pm 16.3$ & $39.9 \pm 14.3$ & 0.9 \\
\hline
\end{tabular}

\begin{tabular}{lccl} 
Table2. Opioids consumption and pain evaluation & & \\
& $\begin{array}{c}\text { PSB group } \\
(\mathrm{n}=32)\end{array}$ & $\begin{array}{c}\text { GA group } \\
(\mathrm{n}=35)\end{array}$ & P value \\
\hline Opioids* consumption & & & \\
Perioperative n (\%) & $15(47 \%)$ & $35(100 \%)$ & $<0.001$ \\
At PACU n (\%) & $9(28 \%)$ & $27(77 \%)$ & $<0.001$ \\
Rescue dose n (\%) & $0(0 \%)$ & $5(14 \%)$ & 0.03 \\
Equivalent fentanyl (mcg) & $25 \pm 30$ & $54 \pm 23$ & $<0.001$ \\
Pain intensity (NRS) : & & & \\
Arrival at PACU & $2.0 \pm 1.8$ & $3.3 \pm 1.4$ & 0.003 \\
Discharge from PACU & $1.6 \pm 1.3$ & $2.3 \pm 1.1$ & 0.01 \\
\hline
\end{tabular}

* opioids included morphine, fentanyl and meperidine

NRS=numeric rating scale

$\mathrm{PACU}=$ post-anesthesia care unit

\begin{tabular}{lccc} 
Table3. Intra-operative events and recovery profile & & \\
\hline & $\begin{array}{l}\text { PSB group } \\
(\mathrm{n}=32)\end{array}$ & $\begin{array}{l}\text { GA group } \\
(\mathrm{n}=35)\end{array}$ & P value \\
\hline $\begin{array}{l}\text { Hemodynamic status arrival PACU } \\
\quad \text { Heart rate (bpm) }\end{array}$ & $71.9 \pm 16$ & $73.8 \pm 17$ & 0.65 \\
$\quad$ Mean arterial pressure (mmHg) & $71.4 \pm 13$ & $74.7 \pm 12$ & 0.46 \\
Adverse events & & & \\
Sore throat (n) & $0(0 \%)$ & $2(6 \%)$ & 0.19 \\
Nausea/Vomiting (n) & $5(16 \%)$ & $15(43 \%)$ & 0.02 \\
Dizziness (n) & $4(13 \%)$ & $9(26 \%)$ & 0.23 \\
Headache (n) & $0(0 \%)$ & $1(3 \%)$ & 0.36 \\
Free from adverse events (n) & $24(75 \%)$ & $21(60 \%)$ & 0.11 \\
Satisfaction & $3.34 \pm 0.5$ & $3.19 \pm 0.8$ & 0.14 \\
\hline
\end{tabular}

$\mathrm{PACU}=$ post-anesthesia care unit

Satisfaction: $1=$ poor, $2=$ fair, $3=$ good, $4=$ very good 